\title{
PERFECT DIFFERENCE SETS
}

\author{
by H. HALBERSTAM and R. R. LAXTON
}

(Received 12 August, 1963)

1. Introduction. If the set $K$ of $r+1$ distinct integers $k_{0}, k_{1}, \ldots, k_{r}$ has the property that the $(r+1) r$ differences $k_{i}-k_{j}(0 \leqq i, j \leqq r, i \neq j)$ are distinct modulo $r^{2}+r+1, K$ is called $a$ perfect difference set mod $r^{2}+r+1$. The existence of perfect difference sets seems intuitively improbable, at any rate for large $r$, but in $1938 \mathrm{~J}$. Singer [1] proved that, whenever $r$ is a prime power, say $r=p^{n}$, a perfect difference set $\bmod p^{2 n}+p^{n}+1$ exists. Since the appearance of Singer's paper several authors have succeeded in showing that for many kinds of number $r$ perfect difference sets mod $r^{2}+r+1$ do not exist; but it remains an open question whether perfect difference sets exist only when $r$ is a prime power (for a comprehensive survey see [2]).

In this note we shall be concerned solely with perfect difference (p.d.) sets $\bmod p^{2 n}+p^{n}+1$, where $p$ is prime. From now on (except in \$2), let $r$ denote $p^{n}$ and write

$$
q=r^{2}+r+1=p^{2 n}+p^{n}+1 \text {. }
$$

We shall lose no generality by assuming that $r>7$.

If $K$ is a p.d. set $\bmod q$ and $K+s$ denotes the set $k_{0}+s, k_{1}+s, \ldots k_{r}+s$ then clearly $K+s$ is also a p.d. set $\bmod q$; since $K$ contains two elements whose difference is congruent to $1(\bmod q)$, there exists a translation $K+s$ which takes these two elements into 0 and 1 . A p.d. set containing 0 and 1 is said to be reduced, and two p.d. sets $\bmod q$ which can be translated to the same reduced set are said to be equivalent.

Singer arrived at his p.d. sets in the following way. Let $G_{3}$ and $G_{1}$ denote respectively the Galois fields $G F\left(p^{3 n}\right)$ and $G F\left(p^{n}\right)$, so that $G_{3}$ is a cubic extension of $G_{1}$. If $\zeta$ is a generator of $G_{3}^{*}$, the multiplicative cyclic group associated with $G_{3}, \zeta$ satisfies a monic cubic equation over $G_{1}$ irreducible in $G_{1}$, and every element of $G_{3}$ can be written in the form

$$
a+b \zeta+c \zeta^{2}, \quad a, b, c \in G_{1}
$$

moreover, every element of $G_{3}$ other than 0 can also be expressed as a power of $\zeta$. Consider then all the elements of $G_{3}$ of the form

$$
a+b \zeta=\zeta^{k}
$$

as $a, b$ run independently through $G_{1}$ but are not both 0 . We say that two such numbers are equivalent if there exists a number $c \neq 0$ in $G_{1}$ such that one is $c$ times the other. The equivalence relation induces a partition of all numbers of the form (1.2) into $r+1$ equivalence classes; for there are, in all, $p^{2 n}-1$ numbers of form (1.2) corresponding to the $p^{2 n}-1$ choices for the pair $a, b$, and on the other hand there are $r-1$ choices for $c$. Let

$$
a_{i}+b_{i} \zeta=\zeta^{k_{1}} \quad(i=0,1, \ldots, r)
$$

be a representative set chosen from these equivalence classes. Then the system $K$ of exponents is a p.d. set $\bmod q$ (a simple proof is given in [3]; see also [4]). A p.d. set constructed in this way will be called a Singer p.d. set, or a p.d. set of Singer type. 
Singer proposed the following two conjectures:

I. All p.d. sets $\bmod p^{2 n}+p^{n}+1$ are of Singer type.

II. There exist exactly $\phi(q) /(3 n)$ reduced Singer p.d. sets.

The chief aim of the present paper is to prove II (see Theorem 2 below). It may be that the method evolved below will be of help in a successful attack on the much more difficult conjecture I.

The main step in the proof of II is Theorem 1 (see \$3), and two proofs of this theorem have appeared recently. One proof is implicit in the results of Bruck [5] and Higman and McLaughlin [6]; the other is Theorem 5 of Gordon, Mills and Welch [7]. The proof given below is different from either of these, and appears to us more elementary in conception.

We are indebted to Dr M. C. R. Butler for a valuable suggestion.

2. The reduction lemma. We begin with a completely elementary result which will provide an essential step in the main argument below (see $\$ 3$ ).

For the purpose of this section we may drop the restriction that $r$ is a prime power.

We say that an integer is written in standard form mod $r^{2}+r+1$ when it is expressed modulo $r^{2}+r+1$ as

$$
u+v r \text { or } u+r^{2} \text { or } r+r^{2}
$$

with integers $u, v$ satisfying

$$
0 \leqq u<r, \quad 0 \leqq v<r .
$$

We say that an integer $t$ is of reduced type mod $r^{2}+r+1$ if

$$
t \equiv u+v r \quad\left(\bmod r^{2}+r+1\right)
$$

where $u, v$ satisfy $(2,2)$ and also

Then

$$
0<u+v \leqq r
$$

LEMMA 1. Let $r$ be a fixed integer greater than 1. Then every integer $t$ greater than 1 and coprime to $r^{2}+r+1$ has the property that $t, t r$ or $t r^{2}$ is of reduced type mod $r^{2}+r+1$.

Proof. If $t \equiv u+r^{2}(\bmod q), 0 \leqq u<r$, then $t r \equiv u r+1(\bmod q)$ and $0<u+1 \leqq r$. If $t \equiv r+r^{2}$, then $t r^{2} \equiv 1+r$ and $0<2 \leqq r$. Thus in the first case $t r$, and in the second $t r^{2}$, are of reduced type $\bmod q$.

It remains to consider the case $t \equiv u+v r(\bmod q), 0 \leqq u, v<r$ and

$$
u+v>r \text {. }
$$

From (2.4) $u+v \geqq r+1$, whence $u=0,1$ is impossible; hence $u \geqq 2$ and similarly $v \geqq 2$.

(i) Suppose that $u=v$. Then $t \equiv u(1+r) \equiv-u r^{2}$; therefore $t r \equiv-u \equiv(r-u)-r$ and $t r^{2} \equiv(r-u) r-r^{2} \equiv(r-u) r+1+r \equiv(r-u+1) r+1$. Hence $t r^{2} \equiv u^{\prime}+v^{\prime} r(\bmod q)$, with $u^{\prime}=1$, $v^{\prime}=r-u+1,0 \leqq u^{\prime}, v^{\prime}<r$ and $u^{\prime}+v^{\prime}=r-u+2 \leqq r$, since $u \geqq 2$. Therefore $t r^{2}$ is of reduced type.

(ii) Suppose that $u>v$. Then $u \geqq v+1$ and

$$
t r \equiv u r+v r^{2} \equiv(u-v) r-v=(u-v-1) r+(r-v) \equiv u^{\prime}+v^{\prime} r(\bmod q),
$$

with $u^{\prime}=r-v, v^{\prime}=u-v-1$ and $0 \leqq u^{\prime}, v^{\prime}<r$. 
If $u^{\prime}+v^{\prime} \leqq r$, then $t r$ is of reduced type. If $u^{\prime}+v^{\prime}>r$, then $r+u-2 v-1>r$, that is, $u>2 v+1$. In this case

$t r^{2} \equiv u r^{2}+v \equiv(v-u)-u r \equiv(v-u)-u r+r^{2}+r+1 \equiv(r+v+1-u)+(r-u) r \equiv u^{\prime \prime}+v^{\prime \prime} r$

$(\bmod q)$,

with $u^{\prime \prime}=r+v+1-u$ and $v^{\prime \prime}=r-u$. Since $u>2 v+1$, we have $0<u^{\prime \prime}, v^{\prime \prime}<r$. Now $u^{\prime \prime}+v^{\prime \prime}=2 r+v+1-2 u>r$ if and only if $r+1+v>2 u$. However, if $u>2 v+1$, then

$$
2 u>2 v+u+1=(v+1)+(v+u)>v+1+r .
$$

It follows that $u^{\prime \prime}+v^{\prime \prime} \leqq r$ and hence $t r^{2}$ is of reduced type.

(iii) Suppose that $v>u$. Then $v \geqq u+1$ and

$t r \equiv u r+v r^{2} \equiv(u-v) r-v \equiv(u-v) r-v+r^{2}+r+1 \equiv(r-v+u) r+(r-v+1) \equiv u^{\prime}+v^{\prime} r(\bmod q)$, with $u^{\prime}=r-v+1, v^{\prime}=r-v+u, 0 \leqq u^{\prime}, v^{\prime}<r$ and $u^{\prime}+v^{\prime}=2 r-2 v+u+1$.

If $u^{\prime}+v^{\prime} \leqq r$, then $t r$ is of reduced type. If $u^{\prime}+v^{\prime}>r$, then $r+u+1>2 v$. In this case, $t r^{2} \equiv u r^{2}+v \equiv(v-u)-u r \equiv(v-u)-u r+r^{2}+r+1 \equiv(r-u+1) r+(v-u+1) \equiv u^{\prime \prime}+v^{\prime \prime} r$

$(\bmod q)$

with $u^{\prime \prime}=v-u+1, v^{\prime \prime}=r-u+1,0 \leqq u^{\prime \prime}, v^{\prime \prime}<r$ and $u^{\prime \prime}+v^{\prime \prime}=r+v-2 u+2$.

If $u^{\prime \prime}+v^{\prime \prime} \leqq r$, then $t r^{2}$ is of reduced type. There remains the case when both $u^{\prime}+v^{\prime}>r$ and $u^{\prime \prime}+v^{\prime \prime}>r$, that is, when $r+u+1>2 v$ and $v+2>2 u$. The first inequality implies that $r+2 u+1 \geqq 2 v+u+1=v+1+(v+u)>v+1+r$, i.e. $2 u \geqq v+1$. This, together with the second inequality, shows that $2 u=v+1$ is the only possibility. Now if $2 u=v+1$ and

$$
r+u+1>2 v=4 u-2,
$$

then $r+3>3 u$. Also $3 u=u+v+1>r+1$ and so we are left with the one case $3 u=r+2$ to consider. But then $3 v=6 u-3=2 r+4-3=2 r+1$ and therefore

$$
3 t \equiv 3 u+3 v r \equiv(r+2)+(2 r+1) r=2\left(r^{2}+r+1\right) \equiv 0(\bmod q)
$$

whence $(t, q)>1$.

3. Multipliers. We need to introduce the notion of a multiplier of a p.d. set (see [2]). Let $t K$ denote the set of integers $t k_{0}, t k_{1}, \ldots, t k_{r}$. If $(t, q)=1$, it is evident that $t K$ is also a p.d. set; we say that $t$ is a multiplier of $K$ if $K$ and $t K$ are equivalent. Clearly, if $t_{1}$ and $t_{2}$ are multipliers, then so is $t_{1} t_{2}$. Singer himself showed in [1] that if $t$ is congruent $\bmod q$ to a power of $p, t$ is a multiplier of any p.d. set of Singer type. (This also follows at once from Lemma 3 in \$4.) The object in this section is to prove the converse (see Theorem 1 below).

We observe that $t$ is a multiplier of $K$ if and only if there exists an integer $s$ such that $t K$ and $K+s$ are identical modulo $q$, i.e. such that for every element $k_{i}$ of $K$ there exists an element $k_{j}$ of $K$ such that

$$
t k_{i} \equiv k_{j}+s(\bmod q)
$$

Bearing in mind the construction of Singer p.d. sets described in $\$ 1$, an equivalent necessary and sufficient condition for $t$ to be a multiplier of the p.d. set of Singer type generated by $\zeta$ is: 
Condition $C$. There exists an integer $s$ with the following two properties: for every $a \in G_{1}$, there exist elements $b, c$ of $G_{1}$ such that

$$
(a+\zeta)^{t}=\zeta^{s}(b+c \zeta)
$$

also, there exist elements $b_{1}, c_{1}$ of $G_{1}$ such that $\zeta^{-s}=b_{1}+c_{1} \zeta$.

We prove

LEMMA 2. Let $t>1$ be an integer of reduced type mod $q$. Then $t$ does not satisfy condition $C$ unless $t$ is congruent mod $q$ to a power of $p$. In particular, $t$ does not satisfy $C$ if $t \equiv u+v r$ and $u+v=r$.

Proof. We may clearly suppose without loss of generality that

$$
1<t<q \text {. }
$$

Let $\dagger$

$$
F(x)=F(x, \zeta)=\prod_{a \in G_{1}}(x-\zeta-a)=x^{r}-x-\left(\zeta^{r}-\zeta\right)
$$

Then we have, modulo $F(x)$, that

$$
x^{r} \equiv x+\zeta^{r}-\zeta \quad \text { and } \quad x^{r^{2}} \equiv x+\zeta^{r^{2}}-\zeta
$$

Further, let

$$
\begin{aligned}
H(x) & =H(x, \zeta)=\prod_{b, c \in G_{1}}\left(x-b \zeta^{s}-c \zeta^{s+1}\right) \\
& =x^{r^{2}}-x^{r \zeta \zeta^{r(r-1) s}}-\left(x^{r}-x \zeta^{(r-1) s}\right)\left(\zeta^{r(s+1)}-\zeta^{r+1}\right)^{r-1},
\end{aligned}
$$

so that

$$
H\left(x^{t}\right)=x^{r^{2} t}-x^{r t \zeta^{r(r-1) s}}-\left(x^{r t}-x^{t \zeta^{(r-1)}}\right)\left(\zeta^{r(s+1)}-\zeta^{r s+1}\right)^{r-1}
$$

is the polynomial having as its zeros the $t$ th roots of all the linear forms $\zeta^{s} b+\zeta^{s+1} c$. Then, by (3.1), $t$ can satisfy the condition $C$ for some $s$ only if

$$
H\left(x^{t}\right) \equiv 0 \quad(\bmod F(x)) .
$$

By (3.2) and (3.3) we have

$$
H\left(x^{t}\right) \equiv\left(x+\zeta^{r^{2}}-\zeta\right)^{t}-A\left(x+\zeta^{r}-\zeta\right)^{t}+B x^{t} \quad(\bmod F(x)),
$$

where

$$
A=\zeta^{r(r-1) s}+\left(\zeta^{r(s+1)}-\zeta^{r s+1}\right)^{r-1}=\zeta^{r(r-1) s}\left(1+\left(\zeta^{r}-\zeta\right)^{r-1}\right),
$$

so that $A \neq 0$, and

$$
B=\zeta^{(r-1) s}\left(\zeta^{r(s+1)}-\zeta^{r s+1}\right)^{r-1}=\zeta^{\left(r^{2}-1\right) s}\left(\zeta^{r}-\zeta\right)^{r-1}
$$

\footnotetext{
+ In the calculations below we make repeated use of the facts that $(x+y)^{p}=x^{p}+y^{p}$ for $x, y \in G_{3}$, and that
$\prod_{a \in G_{1}}(y-a)=y^{r}-y$.
} 
Since $t$ is of reduced type and $t<q$, we may substitute $u+v r$ for $t$ in (3.4) and obtain, after applying (3.2),

$$
\begin{aligned}
0 & \equiv H\left(x^{t}\right)=H\left(x^{u+v r}\right) \\
& \equiv\left(x+\zeta^{r^{2}}-\zeta\right)^{u} x^{v}-A\left(x+\zeta^{r}-\zeta\right)^{u}\left(x+\zeta^{r^{2}}-\zeta\right)^{v}+B x^{u}\left(x+\zeta^{r}-\zeta\right)^{v} \quad(\bmod F(x))
\end{aligned}
$$

The polynomial on the right has degree less than or equal to $u+v$ and so less than or equal to $r$, and the degree of $F$ is $r$. Accordingly, if $u+v=r$, this polynomial and $F$ are essentially the same, and, if $u+v<r$, all the coefficients of the polynomial vanish. This is the situation which we now proceed to exploit. Since $1<u+v r$ and $u+v \leqq r$, we have to consider the following three cases: (i) $u=0$ or $v=0$; (ii) $u>0, v>0, u+v<r$; (iii) $u>0, v>0, u+v=r$.

Case (i). The proof of the lemma in this case has been given in [3]. It can also be proved independently by the methods used below. To be precise, the main result of [3] is that if $t \equiv u, 0<u<r$, then $t$ cannot satisfy $C$ unless it is congruent $\bmod q$ to a power of $p$; and this result also settles the case $t \equiv v r, 0<v<r$.

Case (ii). Since both $u$ and $v$ are positive and $u+v<r$, the constant term in the polynomial on the right of (3.7) must vanish, that is, $A\left(\zeta^{r}-\zeta\right)^{u}\left(\zeta^{r^{2}}-\zeta\right)^{v}=0$. Since none of $A, \zeta^{r}-\zeta$ and $\zeta^{r^{2}}-\zeta$ is 0 , this is impossible. Hence $t$ cannot, in this case, satisfy condition $C$.

Case (iii). Here both $u$ and $v$ are positive and $u+v=r$. If the coefficient of $x^{u+v}\left(=x^{\prime}\right)$ is zero, we refer back to case (ii). If the coefficient of $x^{r}$ is non-zero, the polynomial on the right of (3.7) must be a constant multiple of $F$, and the ratios of the pairs of corresponding coefficients are equal. Since $r>7$ (by hypothesis-see $\$ 1$ ) at least one of $u, v$ exceeds 2; suppose first that both do. Equating the ratios of the coefficients of $x$ and the constant term, we obtain

$$
\frac{1}{a_{1}}=\frac{v}{a_{2}}+\frac{u}{a_{1}}
$$

where $a_{1}=\zeta^{r}-\zeta$ and $a_{2}=\zeta^{2}-\zeta$. It follows that

$$
a_{1}=a_{2}^{r} \text { and } v a_{2}^{(r-1)}=(u-1) \text {. }
$$

Since $a_{2} \neq 0, u \equiv 1(\bmod p)$ if and only if $v \equiv 0(\bmod p)$, and $u+v \equiv 1(\bmod p)$ contradicts $u+v=r$. Hence $u \neq 1(\bmod p), v \neq 0(\bmod p)$ and $p \neq 2$.

We consider the coefficient of $x^{2}$ in (3.7). The coefficient is zero in $F$ since $r>7$; and since $u \geqq 3, v \geqq 3, a_{1} \neq 0, a_{2} \neq 0, p \neq 2$, we have

$$
a_{2}^{2} u(u-1)+2 a_{1} a_{2} u v+a_{1}^{2} v(v-1)=0 .
$$

Applying (3.8), we see that this reduces to $a_{2}^{2(r-1)} v(v-1)=u(u-1)$, and a second application of $(3.8)$ gives $(u-1)((u-1)(v-1)-u v)=0$. But $u \neq 1(\bmod p)$; hence

$$
0 \equiv(v-1)(u-1)-u v=u v-u-v+1-u v=-(u+v-1) \quad(\bmod p) .
$$

Since $u+v=r \equiv 0(\bmod p)$, we have arrived at a contradiction.

It remains to consider the special possibilities

$$
u=1, v=r-1 ; u=2, v=r-2 ; u=r-1, v=1 \text { and } u=r-2, v=2 .
$$


If $u+v r$ is a multiplier, then so is $r(u+v r)$. In the first case

$$
r(u+v r)=r(1+(r-1) r)=r^{3}-r^{2}+r \equiv 2+2 r \quad(\bmod q),
$$

and in the second

$$
r(u+v r)=r(2+(r-2) r)=r^{3}-2 r^{2}+2 r \equiv 3+4 r \quad(\bmod q) .
$$

But from case (ii) above, neither $2+2 r$ nor $3+4 r$ is a multiplier (we recall that $r>7$ ) and so the same can be said of $1+(r-1) r$ and $2+(r-2) r$. If $u+v r$ is a multiplier, then so is $r^{2}(u+v r)$. In the third case

$$
r^{2}(u+v r)=r^{2}((r-1)+r)=2 r^{3}-r^{2} \equiv 2-r^{2} \equiv 3+r \quad(\bmod q),
$$

and in the fourth case

$$
r^{2}(u+v r)=r^{2}((r-2)+2 r)=3 r^{3}-2 r^{2} \equiv 3-2 r^{2} \equiv 5+2 r \quad(\bmod q) .
$$

Again, by case (ii), neither $3+r$ nor $5+2 r$ is a multiplier if $r>7$ and so the same can be said of $(r-1)+r$ and $(r-2)+2 r$.

Hence $t$ cannot, in case (iii), satisfy condition $C$. Thus, to sum up, $t$ can satisfy $C$ only in case (i), and then only when one of $u, v$ is zero and the other is a power of $p$. The proof of the lemma is thus complete.

We are now in a position to prove

THEOREM 1. The only multipliers of perfect difference sets mod $q$ of Singer type are the powers of $p(\bmod q)$.

Proof. It suffices to prove that if $t$ is a multiplier of a p.d. set of Singer type, then $t$ is congruent $\bmod q$ to a power of $p$. By Lemma 2 this is certainly true if $t$ is of reduced type $\bmod q$. Moreover, if $t$ is a multiplier, so is each of $t r, t r^{2}$; and by Lemma 1 , if $t$ is not of reduced type, then at least one of these two must be. The theorem follows at once on appealing again to Lemma 2 .

4. Proof of conjecture II. It remains to prove our main result and, incidentally, to establish another conjecture given in [1], namely, that any two Singer p.d. sets $(\bmod q)$ are connected, i.e. that if $K_{1}, K_{2}$ are two such sets, there exists an integer $t$ such that $K_{1}$ and $t K_{2}$ are equivalent. We require

LEMmA 3. Given a generator $\zeta$ of $G_{3}^{*}$, then, for any integer $t$ coprime with $q$, there exists an integer s such that, for every pair $a, b \in G_{1}$, there exists a pair $c, d \in G_{1}$ such that

$$
a+b \zeta^{t}=\zeta^{s}(c+d \zeta)
$$

Proof. Let

$$
\zeta^{m}=\alpha_{m} \zeta^{2}+\beta_{m} \zeta+\gamma_{m}, \quad \alpha_{m}, \beta_{m}, \gamma_{m} \in G_{1} \quad(m=1,2, \ldots),
$$

and write $\alpha, \beta, \gamma$ for $\alpha_{3}, \beta_{3}, \gamma_{3}$ respectively, so that $\zeta^{3}-\alpha \zeta^{2}-\beta \zeta-\gamma=0$ is the irreducible cubic satisfied by $\zeta$ (see introduction). The $\alpha$ 's, $\beta$ 's and $\gamma$ 's satisfy the following recurrence relations

$$
\alpha_{m+1}=\alpha \alpha_{m}+\beta_{m}, \quad \beta_{m+1}=\beta \alpha_{m}+\gamma_{m}, \quad \gamma_{m+1}=\gamma \alpha_{m} .
$$


We write (4.1) in the form

$$
a+b\left(\alpha_{t} \zeta^{2}+\beta_{t} \zeta+\gamma_{t}\right)=c\left(\alpha_{s} \zeta^{2}+\beta_{s} \zeta+\gamma_{s}\right)+d\left(\alpha_{s+1} \zeta^{2}+\beta_{s+1} \zeta+\gamma_{s+1}\right),
$$

and note that this relation is equivalent to the three simultaneous equations

$$
\begin{aligned}
b \alpha_{t} & =c \alpha_{s}+d \alpha_{s+1}, \\
b \beta_{t} & =c \beta_{s}+d \beta_{s+1}, \\
a+b \gamma_{t} & =c \gamma_{s}+d \gamma_{s+1} .
\end{aligned}
$$

For given $a, b$, these equations are soluble if and only if

$$
\left|\begin{array}{lll}
\alpha_{s} & \alpha_{s+1} & b \alpha_{t} \\
\beta_{s} & \beta_{s+1} & b \beta_{t} \\
\gamma_{s} & \gamma_{s+1} & b \gamma_{t}+a
\end{array}\right|=0
$$

and if $a, b$ now vary over $G_{1}$, this is true only if

$$
\left|\begin{array}{lll}
\alpha_{s} & \alpha_{s+1} & \alpha_{t} \\
\beta_{s} & \beta_{s+1} & \beta_{t} \\
\gamma_{s} & \gamma_{s+1} & \gamma_{t}
\end{array}\right|=0 \text { and } \alpha_{s} \beta_{s+1}-\alpha_{s+1} \beta_{s}=0
$$

and it is easy to check that these two relations determine $\zeta^{s}$ uniquely to within a factor from $G_{1}$.

Lemma 4. $\dagger$ If $K$ is a Singer p.d. set $\bmod q$, and $(t, q)=1$, then $t K$ is also a Singer p.d. set $\bmod q$.

Proof. Suppose that $K$ is generated by $\xi$, a generator of $G_{3}^{*}$, so that

$$
a+b \xi=\xi^{k} \quad(k \in K),
$$

for any pair $a, b \in G_{1}((a, b) \neq(0,0))$. Now solve $\zeta^{t}=\xi$ for $\zeta$, giving another generator of $G_{3}^{*}$. (There is no loss in generality in assuming that $\left(t, r^{3}-1\right)=1$, for $(t, q)=1$ and so $(t+m q, r-1)=1$ for some positive integer $m$ (by Dirichlet's theorem on primes in an arithmetic progression), so that we use $t+m q$ in place of $t$ if $(t, r-1)>1$.) Then (4.2) now reads

$$
a+b \zeta^{t}=\zeta^{t k} \quad(k \in K),
$$

and by Lemma 3 it follows that there exists $s$ such that, for given $a, b \in G_{1}$, there exist $c, d \in G_{1}$ such that $a+b \zeta^{t}=\zeta^{s}(c+d \zeta)$, i.e. we have

$$
\zeta^{i k}=\zeta^{s}(c+d \zeta)
$$

But, on varying $c, d$ over $G_{1}$, this means that $t K-s$ is the p.d. set generated by $\zeta$, i.e. $t K$ is a p.d. set of Singer type.

We mention in passing that Lemma 3 also implies the result to which we referred earlier, namely that every number congruent $\bmod q$ to a power of $p$ is a multiplier of Singer p.d. sets $\bmod q$. To see this we have only to note that if $t \equiv p^{m}(\bmod q),(3.1)$ of condition $C$ reads

$\dagger$ This result is proved in [4] using the theory of projective planes. 


$$
a^{\prime}+\zeta^{t}=\zeta^{s}(b+c \zeta)
$$

the relation discussed in Lemma 3 .

Let $K$ denote a fixed Singer p.d. set $\bmod q$, and let $t$ run through a reduced set of residues $\bmod q$, thereby giving rise to $\phi(q)$ p.d. sets $t K$, each of Singer type by Lemma 4 . By Theorem 1 , these $\phi(q)$ sets fall into $\phi(q) / 3 n$ non-overlapping classes, with $t_{1} K, t_{2} K$ belonging to the same class if and only if $t_{1} \equiv p^{m} t_{2}(\bmod q)$ for some $m$; two of these sets are equivalent or not according as they belong to the same or to different classes. Hence it follows that there exist at least $\phi(q) / 3 n$ non-equivalent p.d. sets $\bmod q$ of Singer type.

In the opposite direction, any Singer p.d. set $\bmod q$ is generated by some generator $\zeta$ of $G_{3}^{*}$, and there exist in all $\phi\left(p^{3 n}-1\right)$ distinct generators of $G_{3}^{*}$ which can be written as $\zeta^{*}$ with $t$ running through a reduced set of residues $\bmod \left(p^{3 n}-1\right)$. However, if $\zeta^{t_{1}}$ and $\zeta^{t_{2}}$ are generators of $G_{3}^{*}$ with $t_{1} \equiv t_{2}(\bmod q), \zeta^{t_{1}}$ and $\zeta^{t_{2}}$ evidently give rise to the same p.d. set; hence we need concern ourselves only with $\phi(q)$ generators $\zeta^{t}$, any two having exponents non-equivalent $\bmod q$. However, if $\zeta^{t_{1}}$ and $\zeta^{t_{2}}$ are two of these generators and $t_{1} \equiv t_{2} p^{m}(\bmod q)$, then $\zeta^{t_{1}}$ and $\zeta^{t_{2}}$ generate equivalent p.d. sets; for if $a+b \zeta^{t_{1}}=\zeta^{t_{1} k}$,

$$
\zeta^{t_{1} k}=a+b^{\prime} \zeta^{t_{2} p^{m}}=\left(a^{\prime \prime}+b^{\prime \prime} \zeta^{t_{2}}\right)^{p^{m}}=\left(\zeta^{t_{2} l}\right)^{p^{m}},
$$

where $/$ runs through the p.d. set generated by $\zeta^{t_{2}}$, and so $\zeta^{t^{1} k}=\zeta^{t_{1} l+d q}$-in other words, $\{k\}$ and $\{l\}$ are equivalent sets. Hence there exist at most $\phi(q) / 3 n$ non-equivalent Singer p.d. sets $\bmod q$. It follows from the previous paragraph that there exist precisely $\phi(q) / 3 n$ non-equivalent Singer p.d. sets $\bmod q$ and that any two of these are connected. We have proved

THEOREM 2. There exist precisely $\phi(q) / 3 n$ reduced Singer p.d. sets mod $q$, any two of which are connected. Two generators $\zeta$ and $\zeta^{t}$ of $G F^{*}\left(p^{3 n}\right)$ give rise to equivalent p.d. sets if and only if $t$ is congruent mod $q$ to a power of $p$.

We remark in conclusion that the reduction lemma (Lemma 1) is relevant to the study of multipliers of p.d. sets mod $r^{2}+r+1$ even when $r$ is not a prime power; in testing whether or not a given $t$ is a multiplier, we know that $t r$ or $t r^{2}$ possesses the same multiplier properties as $t$ and one of $t, t r, t r^{2}$ is of reduced type $\bmod r^{2}+r+1$.

\section{REFERENCES}

1. J. Singer, A theorem of finite projective geometry and some applications to number theory, Trans. Amer. Math. Soc. 43 (1938), 377-385.

2. M. Hall, Jr, A survey of difference sets, Proc. Amer. Math. Soc. 7 (1956), 975-986.

3. H. Halberstam and R. R. Laxton, On perfect difference sets, Quart. J. Oxford Ser. (2) 14 (1963), 86-90.

4. G. Berman, Finite projective plane geometries and difference sets, Trans. Amer. Math. Soc. 74 (1953), 492-499.

5. R. H. Bruck, Difference sets in a finite group. Trans. Amer. Math. Soc. 78 (1955), 464-481. 397.

6. D. G. Higman and J. E. McLaughlin, Geometric $A B A$-groups, Illinois J. Math. 5 (1961), 382-

7. B. Gordon, W. H. Mills and L. R. Welch, Some new difference sets, Canad. J. Math. 14 (1962), $614-625$.

Trinity College, Dublin

UNIVERSITY OF MiCHIGAN and UNIVERSITY OF SUSSEX 